\title{
Professional Competence Evaluation of Information Management Undergraduates Based on Rough Set and D-S Evidence Theory
}

\author{
Di Li*, Hu Wang, Rui Wang and Yazhou Xiong \\ School of Management, Wuhan University of Technology, Wuhan, China \\ lidilee@163.com
}

\begin{abstract}
Based on the analysis of knowledge structure and factors that influence the professional competence of IMIS undergraduates, this paper selects 7 courses as evaluation indexes. Firstly, it gets 3 indexes of database theory, data oriented programming (C\#), Management Information Systems as evidences through the method of information entropy reduction. Secondly, it improves the BPA of subjective experience by using rough set theory and gets an objective BPA. Finally, it uses D-S evidence theory to synthesize the evidence and evaluates the professional competence of IMIS undergraduates.
\end{abstract}

Keywords: professional competence, rough sets, D-S evidence theory, Information Management

\section{Introduction}

With the rapid development of technology, information industry has formed a complete industrial chain. And computer network technology and information technology toward the direction of deepening sophisticated. With the universal use of computers at work and in life, the quality of the whole society in the information system applications, information management, information analysis, information access has been generally improved. This poses a challenge to the major students and requires students to acquire more and better knowledge [1]. Therefore, cultivating students' professional competence has become one of the immediate tasks of undergraduate education in Chinese universities.

As an important element of vocational ability, professional competence is the ability which is relevant to expertise and the ability that can be competent of the job [2]. It is believed that professional competence is interrelated to knowledge, which is indispensable source information of professional competence [3]. Professional competence system consists of three indicators including specialty comprehensive ability, special ability and single ability, and each level indicator is expressed through evaluation objectives, evaluation criteria, evaluation methods, evaluators, evaluation process and evaluation results and so on.

There are many domestic and foreign scholars employing the various methods to evaluate professional competence at present, and the general method to evaluate professional competence is to establish evaluation index system, then index weights and scoring are determined by experts subjectively, finally the total scores are obtained by weighted average. However, there exists no special research on evaluation of the information management undergraduates' professional competence from a quantitative point of view. In this paper, rough set and D-S evidence theory are used to quantitatively evaluate the professional competence. The D-S evidence theory can solve uncertainty of the professional competence evaluation, and when it takes advantage of decision rule strength and decision expansion strength of rough sets theory, can get the less subjective BPA. 


\section{Rough Sets and D-S Evidence Theory}

In this section, the basic concepts of rough sets and D-S evidence theory used in this paper will be briefly discussed.

\subsection{Basic Concepts of Rough Sets Theory}

Rough set theory is a data analysis theory introduced by Polish mathematician Z. Pawlak in 1982. It is a new type of mathematics instrument used to deal with vague and inaccurate problems, as same as a mathematics approach mainly applied to the expression, research and induction of incomplete data or uncertain knowledge [4-5]. Some concepts like decision table, upper and lower approximation, attribute importance are important concepts of rough sets.

Definition 1 Decision table. Let $\mathrm{S}=(\mathrm{U}, \mathrm{A}, \mathrm{V}, \mathrm{f})$ be a decision table. In the decision table, $A=C \cup D$ and $C \cup D=\Phi$, where $C$ is the set of condition attributes and $\mathrm{D}$ is the set of decision attribute. Usually $\mathrm{D}$ contains only one attribute. $\mathrm{U}$ is the universe (a finite set of objects, $\left.\mathrm{U}=\left\{\mathrm{X}_{1}, \mathrm{X}_{2}, \ldots, \mathrm{Xm}\right\}\right),|\mathrm{U}|$ represents the number of objects. $\mathrm{V}$ is the set of attribute values.

Definition Information entropy. The information entropy of knowledge P is:

$$
H(P)=-\sum_{i=1}^{n} p\left(X_{i}\right) \log p\left(X_{i}\right)
$$

Definition 3. Conditional entropy. The conditional entropy that knowledge $P$ respect to knowledge $Q$ is:

In which

$$
\begin{aligned}
& H(Q \mid P)=-\sum_{i=1}^{n} p\left(X_{i}\right) \sum_{j=1}^{m} p\left(Y_{j} \mid X_{i}\right) \log p\left(Y_{j} \mid X_{i}\right) \\
& p\left(Y_{j} \mid X_{i}\right)=\frac{\left|Y_{j} \cap X_{i}\right|}{\left|X_{i}\right|}, i=1,2, \ldots, n, j=1,2, \ldots, m \text { Definition 4. Mutual }
\end{aligned}
$$

information. The mutual information of knowledge $P$ and $Q$ is:

$$
I(P, Q)=H(Q)-H(Q \mid P)
$$

\subsection{Basic Concepts of D-S Evidence Theory}

D-S theory is a generalization of the classical probability theory, which transforms the uncertainty of proposition into the uncertainty of set. It calculations and reasons the elements in $2^{\theta}$, focusing on obtaining BPA (Basic Probability Assignment), belief functions( BeI ), plausibility functions( PI ) and especially evidence combination [6].

Definition 5. BPA. Let $\theta$ be identification framework, if function $\mathrm{m}: 2^{\theta} \rightarrow[0-1]$ satisfying the following conditions: $(1) \mathrm{m}(\Phi)=0 ;(2) \Sigma \mathrm{m}(\mathrm{P})=1$, then $\mathrm{m}(\mathrm{P})$ can be called Basic Probability Assignment. $\mathrm{m}(\mathrm{P})$ stands for the confidence level assigned to the proposition itself, that is the level that support itself occurred, rather than support its proper subset.

\section{The Evaluation Method Based on Rough Sets and D-S Evidence Theory}

The thinking that evaluate the professional competence based on rough sets and D-S theory is as following: one is to preprocess initial historical data and transform data sheet into decision table; two is to reduce attributes based on information entropy; three is to 
obtain BPA based on decision table; four is to combine evidence; and the last is to evaluate professional competence.

(1)Data Preprocessing

The paper summarizes the 7 core courses by analyzing the major's specialty cultivation plan of Chinese key universities. These core courses are oriented programming (C \#), enterprise network engineering, management information systems, MIS database theory, principles of management, data structures, computer program design (C). According to experts, students' academic level divides as follows: 90 points and above is excellent, good is $80-89$ points, $70-79$ points is medium, poor is $60-69$ points, fail is 59 or less. "Excellent" stands for the first rank, "good" for second rank, "medium" for third, "poor" for fourth, "fail" for fifth. Among them, a course corresponds to a condition attribute of decision tables, decision attribute $D$ is the professional ability level. And $d_{l}$ represents excellent professional ability, $d_{2}$ represents good professional ability, $d_{3}$ represents poor.

(2)Attribute Reduction Based on Information Entropy

In general, all kinds of course grades, influenced by environmental factors, may cause conflicts, which means contains a lot of uncertainty. This kind of uncertainty is likely to lead to an increase in conflicts and bring a lot of difficulties to evidence combination. Attribute reduction can reduce this contradiction. In addition, judging professional competence is not have to use all the courses included in specialty cultivation plan, but to choose a group of effective courses, which can reduce information redundancy and calculating load and improve efficiency and effectiveness. On the basis of decision table generated by data preprocessing, this paper reduces attributes using information entropy.

Theorem 1. Let $\mathrm{U}$ be a universe, $P$ is a set of condition attributes, and $d$ is a decision attribute. The necessary and sufficient condition that $r \in P$ is core attribute is:

$$
H(\{d\} \mid P)<H(\{d\} \mid P-\{r\})
$$

In a decision table, we need to consider which attributes are core attributes that can't be got rid of.

(3)Obtain BPA

Many of the current methods are based on the experience or designated by experts to get, which are very subjective. The main idea of this paper is firstly establishing decision table whose condition attributes are evidences(the grades) and the decision attributes are professional ability levels; secondly, calculating the decision rule strength between a single condition attribute and the decision attribute; then, calculating the decision expansion strength; lastly, obtaining BPA by using decision rule strength and decision expansion strength[7].

Theorem 2. Let $P$ be a proposition in identification framework $\theta$, then $P \in 2^{\theta}$. Let $S=(U$, $\mathrm{A}, \mathrm{V}, \mathrm{f}$ ) be a decision table, in which $\mathrm{a} \in 2^{\theta}$.

The numerical range of decision attributes $V_{D}=\theta, H=\{a, D\}, X j \in U / H$, $B_{j}=\left\{H_{i} \mid \bigcup_{H_{i \subset} X_{j}} H_{i}=X_{j}, X_{j} \in U / a\right\},\left|B_{j}\right|=n$, then BPA of $P$ is:

$$
m(P)=m_{1} * m_{2}(P)=\frac{\sum_{i, j B_{i} \cap C_{j}=P} m_{1}\left(B_{i}\right) m_{2}\left(C_{j}\right)}{1-\sum_{i, j B_{i} \cap C_{j}=\phi} m_{1}\left(B_{i}\right) m_{2}\left(C_{j}\right)}
$$




\section{(4) Evidence Combination}

Let us assume $m_{1}$ and $m_{2}$ are two same BPA in identification framework $\theta$, if $\mathrm{BeI}_{1}$ 's focal elements are $B_{1}, B_{2}, \ldots, B_{k}$, and $B e I_{2}$ 's focal elements are $C_{l}, C_{2}, \ldots, C_{n}$. Then Dempster combination rule as below can be applied to evidence combination.

$$
m(P)=\mu_{P} /\left(1+\prod_{i=1}^{n} \mu_{i}\right)
$$

(5)Professional Competence Evaluation

At last, the paper uses the method based on Basic Probability Assignment to judge the level of professional competence. Let us assume existing $P_{1}, P_{2}$ that can satisfy the following conditions.

$$
\left\{\begin{array}{l}
m\left(P_{1}\right)=\max \left\{m\left(P_{i}\right), P_{i} \subset \theta\right\} \\
m\left(P_{2}\right)=\max \left\{m\left(P_{i}\right), P_{i} \subset \theta \text { 且 } P_{i} \neq P_{1}\right\}
\end{array}\right.
$$

If there exists

$$
\left\{\begin{array}{l}
m\left(P_{1}\right)-m\left(P_{2}\right)>\varepsilon_{1} \\
m(\theta)<\varepsilon_{2} \\
m\left(P_{2}\right)>m(\theta)
\end{array}\right.
$$

Then $P_{1}$ is the judgment results, in which $\varepsilon_{1}$ and $\varepsilon_{2}$ are two preset thresholds.

\section{Empirical Study}

The core knowledge structure of information management professionals includes in base layer knowledge, professional layer knowledge, and application layer knowledge. According to training targets and the knowledge structure, there are three kind of abilities required to master, including management ability, technical skills and information capabilities [2]. Combined with the knowledge structure and ability structure, professional competence can be reflected by professional theory courses, professional basic courses, and professional practice courses. This paper uses 7 core courses, including enterprise network engineering, oriented programming $(\mathrm{C} \#)$, principles of management, data structures, computer program design (C), MIS database theory, management information systems, as its condition attributes $C=\left\{x_{1}, x_{2}, x_{3}, x_{4}, x_{5}, x_{6}, x_{7}\right\}$. And $D$ stands for decision attribute, whose numerical range is $\left\{d_{1}, d_{2}, d_{3}\right\}, d_{1}$ represents excellent professional ability, $d_{2}$ represents good professional ability, $d_{3}$ represents poor. The paper uses 181 students' 7 course grades as universe $U$. The professional theory courses contain computer program design (C), principles of management, MIS database theory; the professional basic courses contain data structures, oriented programming $(\mathrm{C} \#)$, enterprise network engineering; and the professional practice course is management information systems.

(1) Data preprocessing

Through data preprocessing, the method we mentioned before, the raw data sheet (Table 1) included the 181 students' 7 course grades is transformed to decision table. And the Table 2 is the decision table after discretization. 
Table 1. Raw Data Sheet

\begin{tabular}{|c|c|c|c|c|c|c|c|c|c|}
\hline$U$ & $x_{1}$ & $x_{2}$ & $x_{3}$ & $x_{4}$ & $x_{5}$ & $x_{6}$ & $x_{7}$ & $D$ & $\begin{array}{l}\text { sample } \\
\text { number }\end{array}$ \\
\hline$r l$ & medium & medium & medium & good & good & good & good & $d 2$ & 33 \\
\hline$r 2$ & poor & medium & good & good & poor & good & good & $d 2$ & 4 \\
\hline$r 3$ & medium & poor & fail & medium & poor & poor & medium & $d 3$ & 5 \\
\hline$r 4$ & excellent & good & good & excellent & excellent & excellent & excellent & $d 1$ & 5 \\
\hline$r 5$ & good & medium & good & excellent & good & good & excellent & $d 2$ & 15 \\
\hline r6 & good & excellent & excellent & good & excellent & excellent & excellent & $d 1$ & 3 \\
\hline$r 7$ & medium & medium & medium & medium & poor & fail & poor & $d 3$ & 4 \\
\hline$r 8$ & good & good & excellent & good & excellent & excellent & excellent & $d 2$ & 23 \\
\hline$r 9$ & excellent & good & good & excellent & excellent & excellent & good & $d 2$ & 11 \\
\hline r10 & excellent & excellent & good & excellent & excellent & good & excellent & $d 1$ & 5 \\
\hline$r 11$ & medium & good & medium & excellent & medium & medium & good & $d 2$ & 35 \\
\hline$r 12$ & excellent & good & good & excellent & good & good & excellent & $d 1$ & 4 \\
\hline$r 13$ & medium & good & medium & good & good & good & excellent & $d 2$ & 18 \\
\hline$r 14$ & medium & good & medium & excellent & medium & poor & good & $d 3$ & 13 \\
\hline$r 15$ & excellent & excellent & excellent & good & excellent & excellent & excellent & $d 1$ & 3 \\
\hline
\end{tabular}

Table 2. Decision Table after Discretization

\begin{tabular}{cccccccccc}
\hline$U$ & $x_{1}$ & $x_{2}$ & $x_{3}$ & $x_{4}$ & $x_{5}$ & $x_{6}$ & $x_{7}$ & $D$ & sample number \\
\hline$r 1$ & 3 & 3 & 3 & 2 & 2 & 2 & 2 & $d 2$ & 33 \\
\hline$r 2$ & 4 & 3 & 2 & 2 & 4 & 2 & 2 & $d 2$ & 4 \\
\hline$r 3$ & 3 & 4 & 5 & 3 & 4 & 4 & 3 & $d 3$ & 5 \\
\hline$r 4$ & 1 & 2 & 2 & 1 & 1 & 1 & 1 & $d 1$ & 5 \\
\hline
\end{tabular}




\begin{tabular}{cccccccccc}
\hline$r 5$ & 2 & 3 & 2 & 1 & 2 & 2 & 1 & $d 2$ & 15 \\
\hline$r 6$ & 2 & 1 & 1 & 2 & 1 & 1 & 1 & $d 1$ & 3 \\
\hline$r 7$ & 3 & 3 & 3 & 3 & 4 & 5 & 4 & $d 3$ & 4 \\
\hline$r 8$ & 2 & 2 & 1 & 2 & 1 & 1 & 1 & $d 2$ & 23 \\
\hline$r 9$ & 1 & 2 & 2 & 1 & 1 & 1 & 2 & $d 2$ & 11 \\
\hline$r 10$ & 1 & 1 & 2 & 1 & 1 & 2 & 1 & $d 1$ & 5 \\
\hline$r 11$ & 3 & 2 & 3 & 1 & 3 & 3 & 2 & $d 2$ & 35 \\
\hline$r 12$ & 1 & 2 & 2 & 1 & 2 & 2 & 1 & $d 1$ & 4 \\
\hline$r 13$ & 3 & 2 & 3 & 2 & 2 & 2 & 1 & $d 2$ & 18 \\
\hline$r 14$ & 3 & 2 & 3 & 1 & 3 & 4 & 2 & $d 3$ & 13 \\
\hline$r 15$ & 1 & 1 & 1 & 2 & 1 & 1 & 1 & $d 1$ & 3 \\
\hline
\end{tabular}

(2)Attribute Reduction

According to rough set theory, we can get:

$$
\begin{aligned}
& U / C=\left\{r_{1}, r_{2}, r_{3}, r_{4}, r_{5}, r_{6}, r_{7}, r_{8}, r_{9}, r_{10}, r_{11}, r_{12}, r_{13}, r_{14}, r_{15}\right\} \\
& U / D=\left\{\left(r_{4}, r_{6}, r_{10}, r_{12}, r_{15}\right),\left(r_{1}, r_{2}, r_{5}, r_{8}, r_{9}, r_{11}, r_{13}\right),\left(r_{3}, r_{7}, r_{14},\right)\right\}
\end{aligned}
$$

According to Formula (1):

$$
H(D)=-\left(\frac{5}{15} \log _{2} \frac{5}{15}+\frac{7}{15} \log _{2} \frac{7}{15}+\frac{3}{15} \log _{2} \frac{3}{15}\right)=1.506
$$

According to Formula (2) :

$$
\begin{gathered}
H(D \mid C)=-\left(\frac{1}{15}\left(0+1 \log _{2} 1+0\right)+\frac{1}{15}\left(0+1 \log _{2} 1+0\right)+\frac{1}{15}\left(0+0+1 \log _{2} 1\right)\right. \\
+\frac{1}{15}\left(1 \log _{2} 1+0+0\right)+\frac{1}{15}\left(0+1 \log _{2} 1+0\right)+\frac{1}{15}\left(1 \log _{2} 1+0+0\right)+\frac{1}{15}\left(0+0+1 \log _{2} 1\right) \\
+\frac{1}{15}\left(0+1 \log _{2} 1+0\right)+\frac{1}{15}\left(0+1 \log _{2} 1+0\right)+\frac{1}{15}\left(1 \log _{2} 1+0+0\right)+\frac{1}{15}\left(0+1 \log _{2} 1+0\right) \\
+\frac{1}{15}\left(1 \log _{2} 1+0+0\right)+\frac{1}{15}\left(0+1 \log _{2} 1+0\right)+\frac{1}{15}\left(0+0+1 \log _{2} 1\right)+\frac{1}{15}\left(1 \log _{2} 1+0+0\right)=0
\end{gathered}
$$

According to Formula $(3): I(C, D)=H(D)-H(D \mid C)=1.506$

In the same way: $I\left(C-\left\{x_{1}\right\}, D\right)=1.506, I\left(C-\left\{x_{2}\right\}, D\right)=1.373$,

$$
\begin{aligned}
& I\left(C-\left\{x_{3}\right\}, D\right)=1.506, I\left(C-\left\{x_{4}\right\}, D\right)=1.506, \quad I\left(C-\left\{x_{5}\right\}, D\right)=1.506, \\
& I\left(C-\left\{x_{6}\right\}, D\right)=1.373, \quad I\left(C-\left\{x_{7}\right\}, D\right)=1.373 \\
& I(C, D)>I\left(C-\left\{x_{2}\right\}, D\right), I(C, D)>I\left(C-\left\{x_{6}\right\}, D\right), I(C, D)>I\left(C-\left\{x_{7}\right\}, D\right)
\end{aligned}
$$

Therefore, $x_{2}, x_{6}, x_{7}$ are core attributes, and the core attribute set $C_{0}=\left\{x_{2}, x_{6}, x_{7}\right\}$. Let $B=C_{0}, I(B, D)=1.373$ can be obtained by calculating. Because of $I(B, D)=I(\mathrm{C}, D), B$ is relative reduction to decision Table 1 , that is relative reduction $B=\left\{x_{2}, x_{6}, x_{7}\right\}$ is core courses. We can get Table 2 after getting rid of attributes not belonging to $B$. 
Table 3. Decision Table after Reduction

\begin{tabular}{cccccc}
\hline$U$ & $x_{2}$ & $x_{6}$ & $x_{7}$ & $D$ & sample number \\
\hline$r 1$ & 3 & 2 & 2 & $d 2$ & 33 \\
\hline$r 2$ & 3 & 2 & 2 & $d 2$ & 4 \\
\hline$r 3$ & 4 & 4 & 3 & $d 3$ & 5 \\
\hline$r 4$ & 2 & 1 & 1 & $d 1$ & 5 \\
\hline$r 5$ & 3 & 2 & 1 & $d 2$ & 15 \\
\hline$r 6$ & 1 & 1 & 1 & $d 1$ & 3 \\
\hline$r 7$ & 3 & 5 & 4 & $d 3$ & 4 \\
\hline$r 8$ & 2 & 1 & 1 & $d 2$ & 23 \\
\hline$r 9$ & 2 & 1 & 2 & $d 2$ & 11 \\
\hline$r 10$ & 1 & 2 & 1 & $d 1$ & 5 \\
\hline$r 11$ & 2 & 3 & 2 & $d 2$ & 35 \\
\hline$r 12$ & 2 & 2 & 1 & $d 1$ & 4 \\
\hline$r 13$ & 2 & 2 & 1 & $d 2$ & 18 \\
\hline$r 14$ & 2 & 4 & 2 & $d 3$ & 13 \\
\hline$r 15$ & 1 & 1 & 1 & $d 1$ & 3 \\
\hline
\end{tabular}

\section{(3)Obtain BPA}

Given a student's grade:(2,3,3,2,2,2,2), that means enterprise network engineering is good, oriented programming $(\mathrm{C} \mathrm{\#})$ is medium, principles of management is medium, data structures is good, computer program design (C) is good, MIS database theory is good, and management information systems is also good.

For course $\mathrm{x}_{2}$ :

$U /\left\{\mathrm{x}_{2}, D\right\}=\left\{\left\{r_{4}, r_{12}\right\},\left\{r_{6}, r_{10}, r_{15}\right\},\left\{r_{8}, r_{9}, r_{11}, r_{13}\right\},\left\{r_{1}, r_{2}, r_{5}\right\},\left\{r_{3}\right\},\left\{r_{7}\right\},\left\{r_{14}\right\}\right\}$

$U / \mathrm{x}_{2}=\left\{\left\{r_{6}, r_{10}, r_{15}\right\},\left\{r_{4}, r_{8}, r_{9}, r_{11}, r_{12}, r_{13}, r_{14}\right\},\left\{r_{1}, r_{2}, r_{5}, r_{7}\right\},\left\{r_{3}\right\}\right\}$

The course $\mathrm{x}_{2}$ is 3 , so $B_{3}=\left\{\left\{r_{1}, r_{2}, r_{5}\right\},\left\{r_{7}\right\}\right\}$

At first, calculating the decision rule strength of $\left\{r_{1}, r_{2}, r_{5}\right\}$ to get $\mu_{I}=(33+4+15) /(33+4+15+8)=0.87$; secondly, calculating the decision rule strength of $\left\{r_{7}\right\}$ to get $\mu_{2}=8 /(33+4+15+8)=0.13$; at last, calculating the decision rule strength $\mu_{3}=\mu_{1}^{*} \mu_{2}=0.1131$.

According to Formula(4), getting the following evidences:

$m_{x 2}\left(d_{2}\right)=0.7816, m_{x 2}\left(d_{3}\right)=0.1168, m_{x 2}\left(d_{2}, d_{3}\right)=0.1016$.

For course $\mathrm{x}_{6}$ :

$$
\begin{aligned}
& U /\left\{\mathrm{x}_{6}, D\right\}=\left\{\left\{r_{4}, r_{6}, r_{15}\right\},\left\{r_{10}, r_{12}\right\},\left\{r_{1}, r_{2}, r_{5}, r_{13}\right\},\left\{r_{8}, r_{9}\right\},\left\{r_{11}\right\},\left\{r_{3}, r_{14}\right\},\left\{r_{7}\right\}\right\} \\
& U / \mathrm{x}_{6}=\left\{\left\{r_{4}, r_{6}, r_{8}, r_{9}, r_{15}\right\},\left\{r_{1}, r_{2}, r_{5}, r_{10}, r_{12}, r_{13}\right\},\left\{r_{11}\right\},\left\{r_{3}, r_{14}\right\},\left\{r_{7}\right\}\right\}
\end{aligned}
$$

The course $x_{6}$ is 3 , so

$$
B_{2}=\left\{\left\{r_{1}, r_{2}, r_{5}, r_{13}\right\},\left\{r_{10}, r_{12}\right\}\right\}
$$


At first, calculating the decision rule strength of $\left\{r_{1}, r_{2}, r_{5}, r_{13}\right\}$ to get $\mu_{I}=(33+4+15+18) /(33+4+15+18+5+4)=0.886$; secondly, calculating the decision rule strength of $\left\{r_{10}, r_{12}\right\}$ to get $\mu_{2}=(5+4) /(33+4+15+18+5+4)=0.114$; at last, calculating the decision rule strength $\mu_{3}=\mu_{1}^{*} \mu_{2}=0.101004$.

According to Formula (4), getting the following evidences:

$$
m_{x 6}\left(d_{1}\right)=0.1036, m_{x 6}\left(d_{2}\right)=0.8047, m_{x 2}\left(d_{1}, d_{2}\right)=0.0917 \text {. }
$$

For course $\mathrm{x}_{7}$ :

$$
\begin{aligned}
& U /\left\{\mathrm{x}_{7}, D\right\}=\left\{\left\{r_{4}, r_{6}, r_{10}, r_{12}, r_{15}\right\},\left\{r_{1}, r_{2}, r_{9}, r_{11}\right\},\left\{r_{5}, r_{8}, r_{13}\right\},\left\{r_{3}\right\},\left\{r_{7}\right\},\left\{r_{14}\right\}\right\} \\
& U / \mathrm{x}_{7}=\left\{\left\{r_{4}, r_{5}, r_{6}, r_{8}, r_{10}, r_{12}, r_{13}, r_{15}\right\},\left\{r_{1}, r_{2}, r_{9}, r_{11}, r_{14}\right\},\left\{r_{3}, r_{7}\right\}\right\}
\end{aligned}
$$

The course $x_{7}$ is 2 , so

$$
B_{2}=\left\{\left\{r_{1}, r_{2}, r_{9}, r_{11}\right\},\left\{r_{14}\right\}\right\}
$$

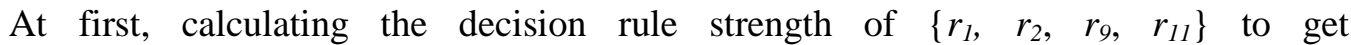
$\mu_{1}=(33+4+11+35) /(33+4+11+35+13)=0.865$; secondly, calculating the decision rule strength of $\left\{r_{14}\right\}$ to get $\mu_{2}=13 /(33+4+15+35+13)=0.116$; at last, calculating the decision rule strength $\mu_{3}=\mu_{1} * \mu_{2}=0.10034$.

According to Formula (4), getting the following evidences:

$$
m_{x 7}\left(d_{2}\right)=0.8291, m_{x 7}\left(d_{3}\right)=0.09, m_{x 7}\left(d_{2}, d_{3}\right)=0.8108 \text {. }
$$

(4)Evidence Combination

According to Formula (5), this paper combines course $\mathrm{x}_{2}, \mathrm{x}_{6}, \mathrm{x}_{7}$ and gets:

$$
m\left(d_{1}\right)=0, m\left(d_{2}\right)=1, m\left(d_{3}\right)=0, m\left(d_{1}, d_{2}\right)=0, m\left(d_{2}, d_{3}\right)=0, m\left(d_{1}, d_{2}, d_{3}\right)=0
$$

(5)Professional Competence Rating

According to Formula (6) and Formula (7), $m\left(P_{1}\right)-m\left(P_{2}\right)=m\left(d_{2}\right)-m\left(d_{1}\right)=1$.

The difference between adjacent BPA is far greater than $\varepsilon 1$ and $\mathrm{m}(\theta)=0$ is far smaller than $\varepsilon 2$. Therefore, the professional competence level is $d 2$ and the method is effective. Compared with decision table after reduction, we can know the example we taken conforms to $r l$. In summary, we can judge the professional competence level is $d 2$, which is good.

\section{Conclusions}

On the basis of the evaluation model and the empirical study, the following conclusion can be drawn:

As we know, basic probability assignment of D-S theory is subjective, but this paper calculates BPA based on decision tables probability of rough set theory, which objectively reflects the confidence of the proposition. The three core courses that derived by attributes reduction, including oriented programming (C \#), MIS database theory, management information systems can reflect the influence of professional knowledge level to professional competence. The organic combination of rough set theory and D-S evidence theory both has enhanced the ability to deal with uncertain decision problems, and has effectively determined students' professional competence levels.

As a comprehensive practical application professional core curriculum, Management Information System is based on the professional knowledge and skills which are cultivated in the professional theory and basic courses, and has developed undergraduates' comprehensive application of management science and computer software programming. Secondly, as the fuse characteristic curriculum, oriented programming $(\mathrm{C} \#)$ has distinguished information management from other management majors, which has a great effect on students' professional competence. In addition, as a professional theory course, database theory lays a solid foundation for specialty system. To a large extent, these 3 core courses can embody the curriculum system of Information 
Management major and cultivate students' core competencies, as well as professional competence.

This evaluation method can not only be used for evaluating Information Management students' professional competence, but also can guide reform of Information Management students' curriculum system, promoting the cultivation of university students' autonomous learning, practice and innovation to meet the needs of the society for Information Management students' professional competence.

\section{Acknowledgements}

This paper is supported by Hubei provincial education department, Teaching research project (2012113, 2013108), Fundamental Research Funds for the Central Universities No. WHUT 2014zy-042, the in-depth business plan of action funded projects of the 2014 Hubei Provincial College Yong Teachers under Grant No.XD2014668 and Social Science Research Project of the 2014 Hubei Provincia Department of Education Grant No.14Y069.

\section{References}

[1] Y. Li and G. Xiaojun, "Research on innovation ability cultivation of information management Professionals", Modern Information, no.6, (2012), pp. 139-141.

[2] W. Yong, M. Hai and C. Jianyong, "Construct undergraduates' curriculum system of Information management based on professional ability cultivation", Computer education, no.2, (2012), pp. 10-13,17.

[3] H. Yongang and H. Lihua, "Summary on information management curriculum", Information Journal, no. 8, (2007), pp. 128-131.

[4] Z. Pawlak, "Why rough sets", Pro. of the Fifth IEEE International Conference on Fuzzy Systems, Orleans, vol. 2, no. 1, (1996), pp. 738-743.

[5] Z. Pawlak, "Rough sets", International Journal of Information and Computer Science, vol. 11, no. 1, (1982), pp. 341-356.

[6] C. Chao, C. Xingyuan, Y. Yinjie and W. Yongwei, "Security assessment system based on rough set theory and D-S evidence", Computer Engineering, no. 10, (2013), pp. 138-142.

[7] L. Yanli, L. Yingjie and W. Jinjin, "Identity fusion method based on rough D-S theory", Systems Engineering and Electronics, no. 10, (2007), pp. 1749-1752.

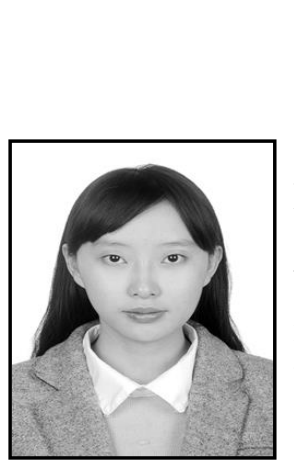

\section{Authors}

Di Li, received her Management Bachelor's degree in School of Management at Wuhan University of Technology in 2013. Currently, she is now studying $\mathrm{PhD}$ in Management science and Engineering at Wuhan University of Technology. Her current research interests include different aspects of High-level scientific and technological talents, Service Mining.

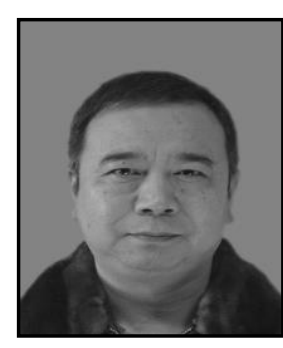

Hu Wang, is a Professor in School of Management at Wuhan University of Technology. Now he is the head of Information Management Department at Wuhan University of Technology. His current research interests include different aspects of Enterprise informatization, Service Mining, Knowledge Management. 


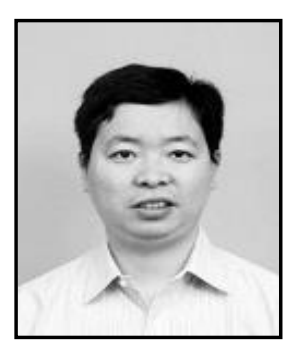

Rui Wang, obtained the M.S. Degree in Management Science and Engineering from Wuhan University of Technology. Now, he has received $\mathrm{PhD}$ doctor's degree in in Management science and Engineering at Wuhan University of Technology. His current research interests include different aspects of information management, E-commerce.

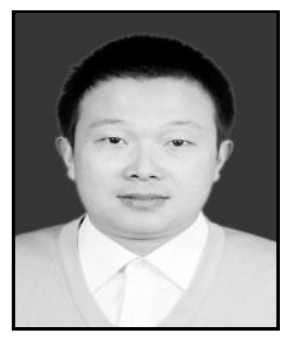

Yazhou Xiong, obtained the M.S. Degrees in Management Science and Engineering from Wuhan University of Technology, China, in 2005. He is an associate professor, $\mathrm{PhD}$ candidate. 